\title{
Method for assessing the dynamics and efficiency of diving fins
}

\author{
ANDRZEJ GRZĄDZIELA ${ }^{1}$, PIOTR SZYMAK ${ }^{2}$, PAWEŁ PISKUR ${ }^{2}$ \\ ${ }^{1}$ Institute of Construction and Operation of Vessels, Faculty of Mechanical and Electrical Engineering, \\ Polish Naval Academy, Gdynia, Poland. \\ ${ }^{2}$ Institute of Electrical Engineering and Automatics, Faculty of Mechanical and Electrical Engineering, \\ Polish Naval Academy, Gdynia, Poland.
}

\begin{abstract}
This article presents a method for an evaluation of the dynamic ability and efficiency of diving fins. There is paucity in the literature on the process of selecting optimal fins. As a result, there are efforts made to develop a methodology for selecting fins that meet the proposed criteria. In the present study, an analysis on the two types of fins most popular within the commercial market was conducted. The experiment took place in a test water tunnel fully equipped with a measuring system and strain gauges for recording forced interaction between the moving fin and flowing water. The tested fins rested on an artificial leg, which moved respectively, thereby developing movement algorithms. This forced fluid flow was implemented by a pump that was able to control the fluids velocity, and a non-invasive method involving an ultrasonic flow meter was used to measure the fluids velocity. Finally, the fin efficiency was calculated as the ratio of multiplication of generated thrust to electrical energy consumption whilst also considering the mechanical efficiency of the leg manipulator. The results of these experiments are discussed in depth and a method is created for the subsequent stage in which a new type of fins called biomimetic is to be analyzed and compared.
\end{abstract}

Key words: the efficiency of diving fins, water tunnel, manipulator of diver's leg

\section{Introduction}

The commercial diving is a popular way of recreation, and it is growing every year. Diving has a variety of tasks ranging from sports and recreation to the exploration of wrecks or studying of marine fauna and flora. The diving equipment market is currently extensive, and the customer has various equipment offered by many companies at his disposal. Currently produced fins allow for three basic modes of propulsive finning, i.e., scissor (flutter kick), frog kick and dolphin kick. This paper focused on the energetic analysis of the fins of the flutter kick mode types. Most fin performance tests refer to medical examinations for oxygen consumption under the same swim conditions [19], or they are focused on increasing a swimming speed or an efficiency received by the swimmers and/or divers [5], [8]. However, the presented analyses are ambiguous because the oxygen consumption depends on metabolic processes, physical condition, training status and neuromuscular factor on the day of diving. Difficulties in assessing fin parameters are mainly caused by a strongly nonlinear interaction between flexible fin and fluid. Moreover, this interaction is currently possible to simulate only for a discrete model, with assumed a number of degrees of freedom [8]. Even if any laboratory test stand is designed and used for fin parameter experimental verification, the results have not been published. Parameters and production details are highly guarded trade secrets. Some results made by scientific teams can be found in [21], but all tests were provided for the human body, with re-

\footnotetext{
* Corresponding author: Andrzej Grządziela, Institute of Construction and Operation of Vessels, Faculty of Mechanical and Electrical Engineering, Polish Naval Academy. Phone: 501120890, e-mail: a.grzadziela@amw.gdynia.pl

Received: March 9th, 2020

Accepted for publication: October 19th, 2020
} 
(a)



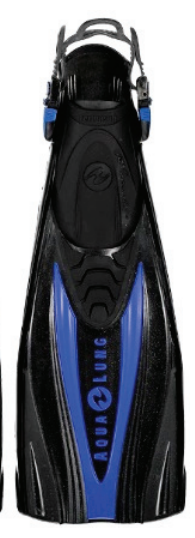

(b)

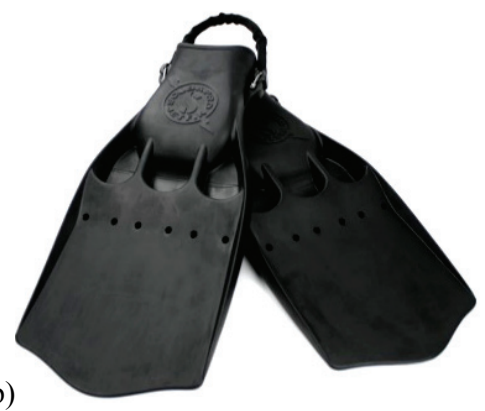

(c)

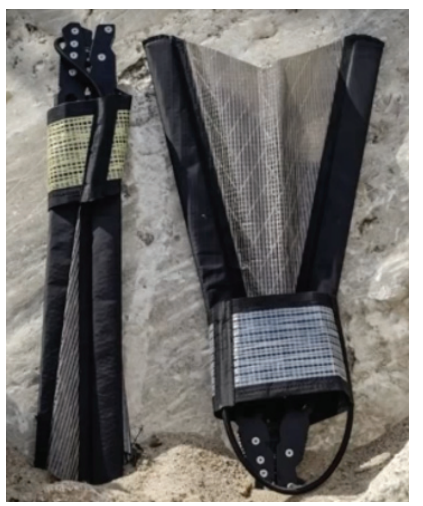

Fig. 1. Swimmer's fins Express from Aqualung company (a), diver's fins Jet Fin from Scubapro company (b), fins from Exotech company for military purposes (c)

striction mention above. The experiment presented here was made for the artificial leg with defined control algorithms. The designed software can archive all the input and output data for the objective assessment of fin parameters and coefficient. In Figures 1a, b, two reference fins used for the designed methodology verification are depicted. Then, the newly designed biomimetic fins (Fig. 1c) were compared with the tested ones. The process of implementation of the new biomimetic fins for civilian purposes is in progress. In addition to the laboratory test stand, additional tests were carried out among professional divers.

This paper was organized as follows. In Section 2.1, the geometry of the test tunnel with equipment for water velocity control is described. The design of the artificial leg and its implementation in the water tunnel was dis- cussed in Section 2.2. Section 2.3 provided a discussion on the leg kinematics and dynamics. Chapter 2.4 includes the description of plan the experiment and software used as well. Finally, the test results achieved according to the proposed methodology are presented in Chapter 3, and the conclusions and future research are depicted in Chapter 4.

\section{Materials and methods}

\subsection{The water tunnel}

The tunnel was made of laminate parts, with dimensions depicted in Fig. 2. In Figure 3, the photography of
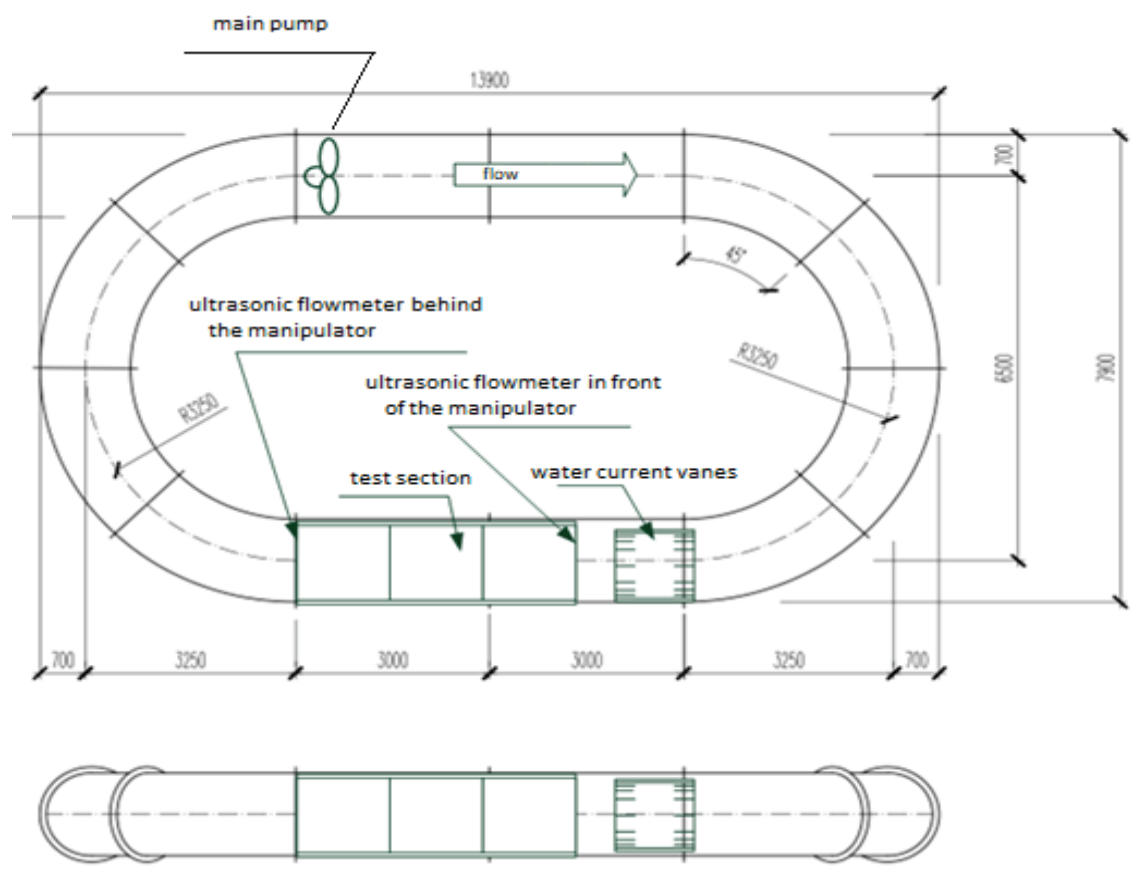

Fig. 2. Geometry of the water tunnel 


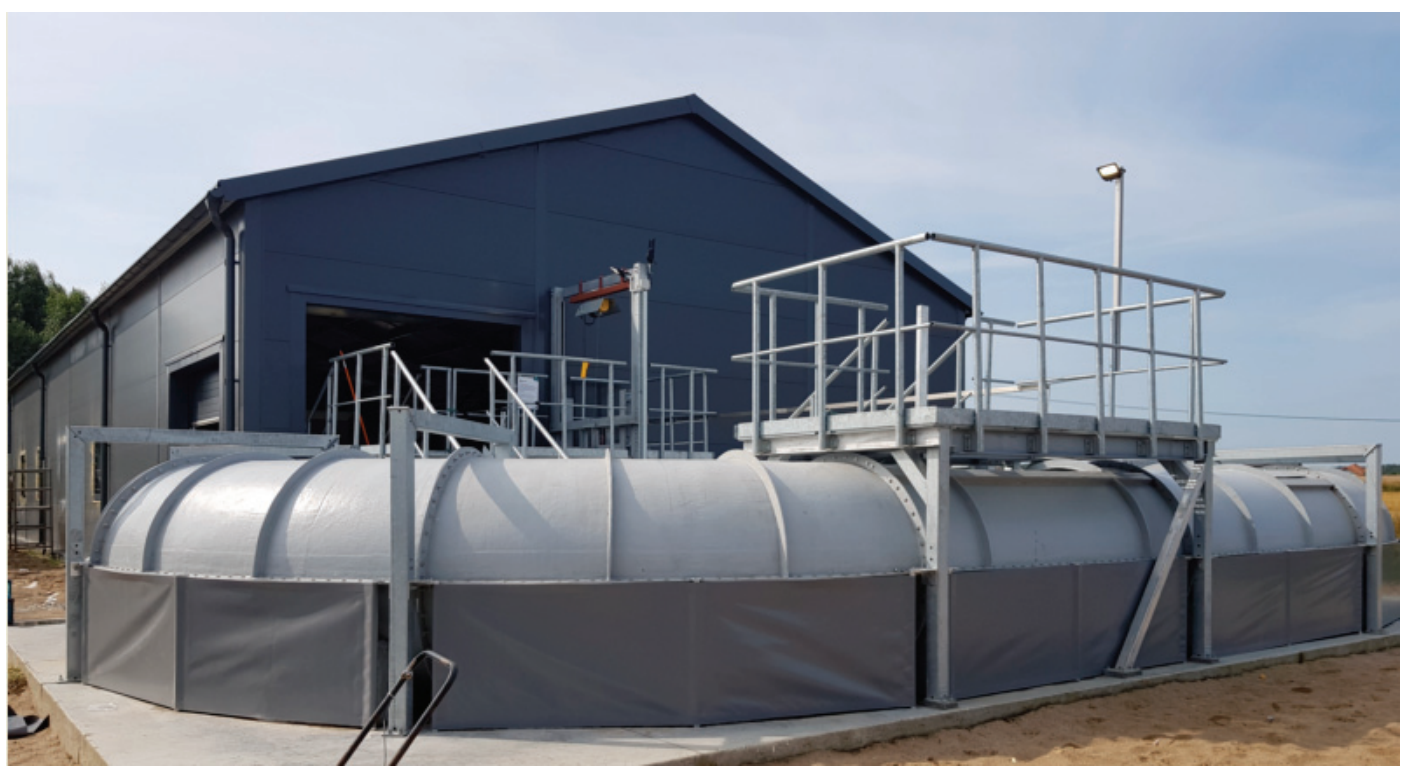

Fig. 3. Photography of the water tunnel

the water tunnel after construction is presented. It is an open type channel with additional remote-control water pump for keeping the desired fluid velocity [14]. The fluid speed was measured with the specialised ultrasonic flowmeter. Besides, the vanes were placed in front of the manipulator's leg to maintain laminar flow. The interaction between the leg with fin and fluid was measured with strain gauge system mounted inside manipulator. Plexiglas window was mounted in the test section for observing and measuring the vertical strokes of moving fins. Above the test section, a crane was mounted for submersion of the manipulator in the tunnel.

\subsection{The leg's manipulator}

The diver's body was divided into anatomical, movable segments, while the horizontal position of the torso with hands and head was assumed, acting as a support [18]. A general view of the manipulator leg design is shown in Fig. 4a. The more detailed cross-section with kinematic structure and dimensions is depicted in Fig. 4b. The moving elements were the thigh, lower leg and foot with the fin. Passive video recording occurred on markers attached to the diver at the following points: fin trailing edge, calcaneus of the foot, the lateral malleolus of the ankle, the lateral condyle of the knee and the greater trochanter of the hip [2], [8]. The diver's leg movement was recorded in the pool with a Panasonic camera, set on a tripod, recording videos at 30 fps. Reflective markers were illuminated with artificial light and the analysis included movements in twodimensional coordinates.

The biomechanical study of leg movement involved the Cartesian system where the centre of the coordi- (a)

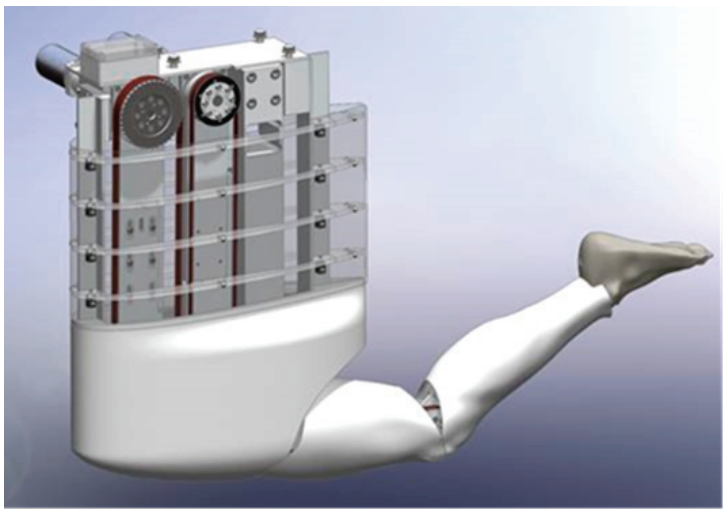

(b)

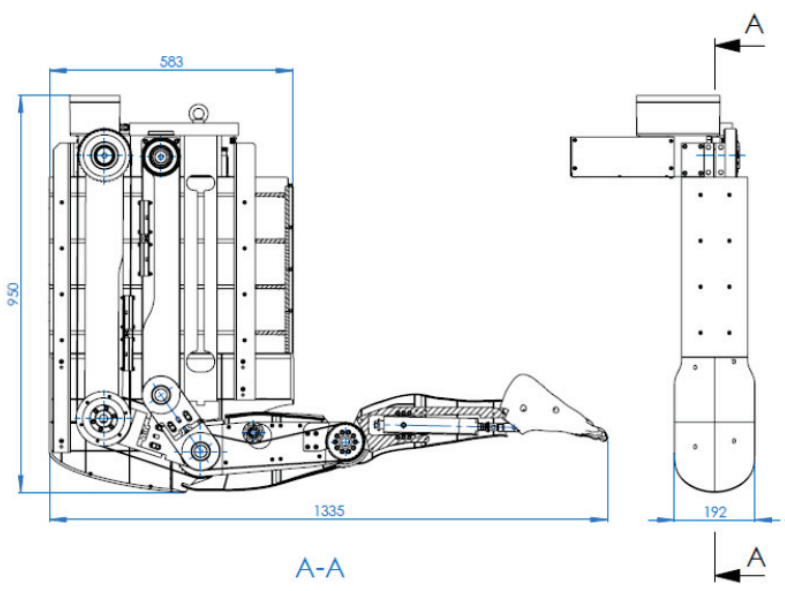

Fig. 4. The leg's manipulator: design (a), cross-section with dimensions (b) 
(a)

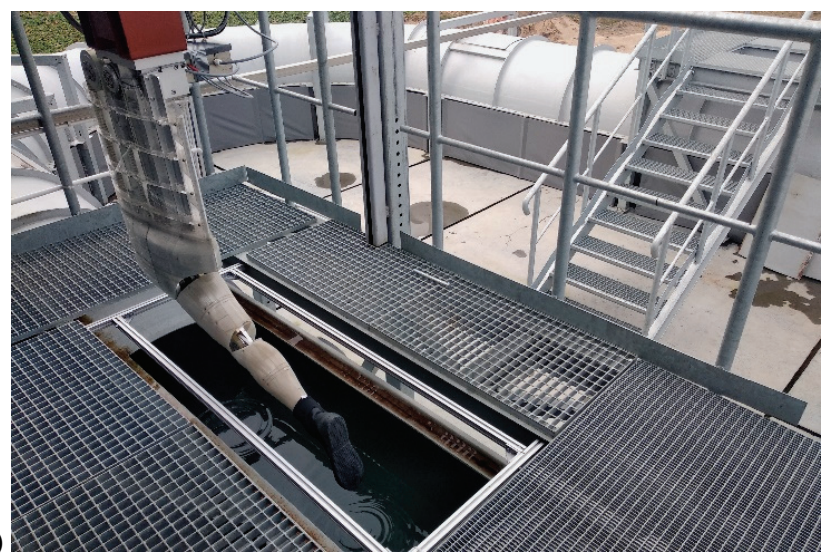

(b)

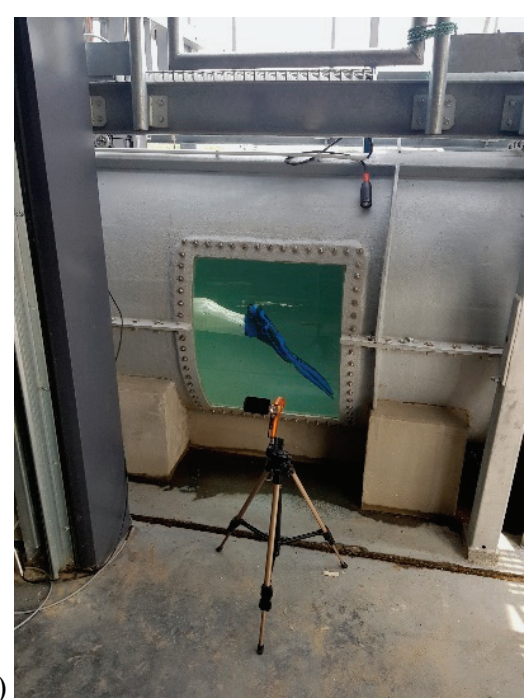

Fig. 5. Manipulator leg: above the surface of the water tunnel (a), in the Plexiglas window during reference fin testing (b)

nate system was in the greater trochanter of the hip. Leg displacement was considered to be a progressive change in the position of the markers over time, hence, it was considered a vector. The calculations of the marker displacement are shown in $Y-0-X$ coordinates and then calculated as a resultant vector from the Pythagorean relationship. Velocity vectors were also obtained from the relative differences of displacements and the time of their positions. A sample of velocity measures is collected in the table's vs time diagram, which enabled acceleration tables for all markers to be obtained in a similar way. The research results allowed the presentation of different slopes of all tags versus time. The leg's manipulator above the surface of the water tunnel just before immersion is shown in Fig. 5a. In Figure 5b, the manipulator leg with the referenced fin is visible through a Plexiglas window. The photo was taken during the test and video recording for further kinematics and dynamic analysis for each fin being examined.

The manipulator allows for the simulation of the diver's leg movement in the range of deflections (amplitudes) and movement frequencies adopted as characteristic for recreational swimming. The device is equipped with two drive motors powered by DC voltage $U=24 \mathrm{~V}$, making cyclic movements of the thigh and lower leg, simulating the movement of the diver's leg. The measuring system enables precise current readings drawn by both motors with a sampling frequency $f_{s}=1 \mathrm{kHz}$. This solution allows for reading of current values, which, at the known supply voltage, allows for the calculation of instantaneous power demand values.

\subsection{Kinematics and dynamics of the leg with fin}

The recreational swimming speeds were taken into consideration to analyze the energy efficiency of two types of fins. Speeds for professional sport swimming in the fins reach up to $0.92 \mathrm{~m} / \mathrm{s}$, while recreational swimming and exploration are the speed range from 0.2 to $0.3 \mathrm{~m} / \mathrm{s}$. The method for the selection of the kinematic parameters of the manipulator's leg movement has been developed using the results of studies presented in the literature [11], [17]. At the initial stage of the project, tests were carried out in the pool to determine the typical movements of the diver's leg during recreational swimming. The developed operational scenarios included free swimming, with low speeds, rapid acceleration and rapid immersion. For all scenarios, visual analysis of the range of motion and frequency of hip, thigh, lower leg and two tags of diver's feet were developed. Due to the different fin lengths, the parameters analyzed were the displacement of the knee and the ankle of the swimmer's, respectively. The ankle joint is reflected in the form of a flexible element with a limited swing angle, determined in the indicated tags of an ankle and toes measurements. Taking under consideration the manipulator kinematics and the need to change the dynamics depending on the type of fins, the following parameters (Fig. 6) were used to control the leg:

- P1 - the lower position of the lower leg,

- $\mathrm{P} 2$ - the target upper thigh position,

- P3 - the angular velocity of thigh movement, 

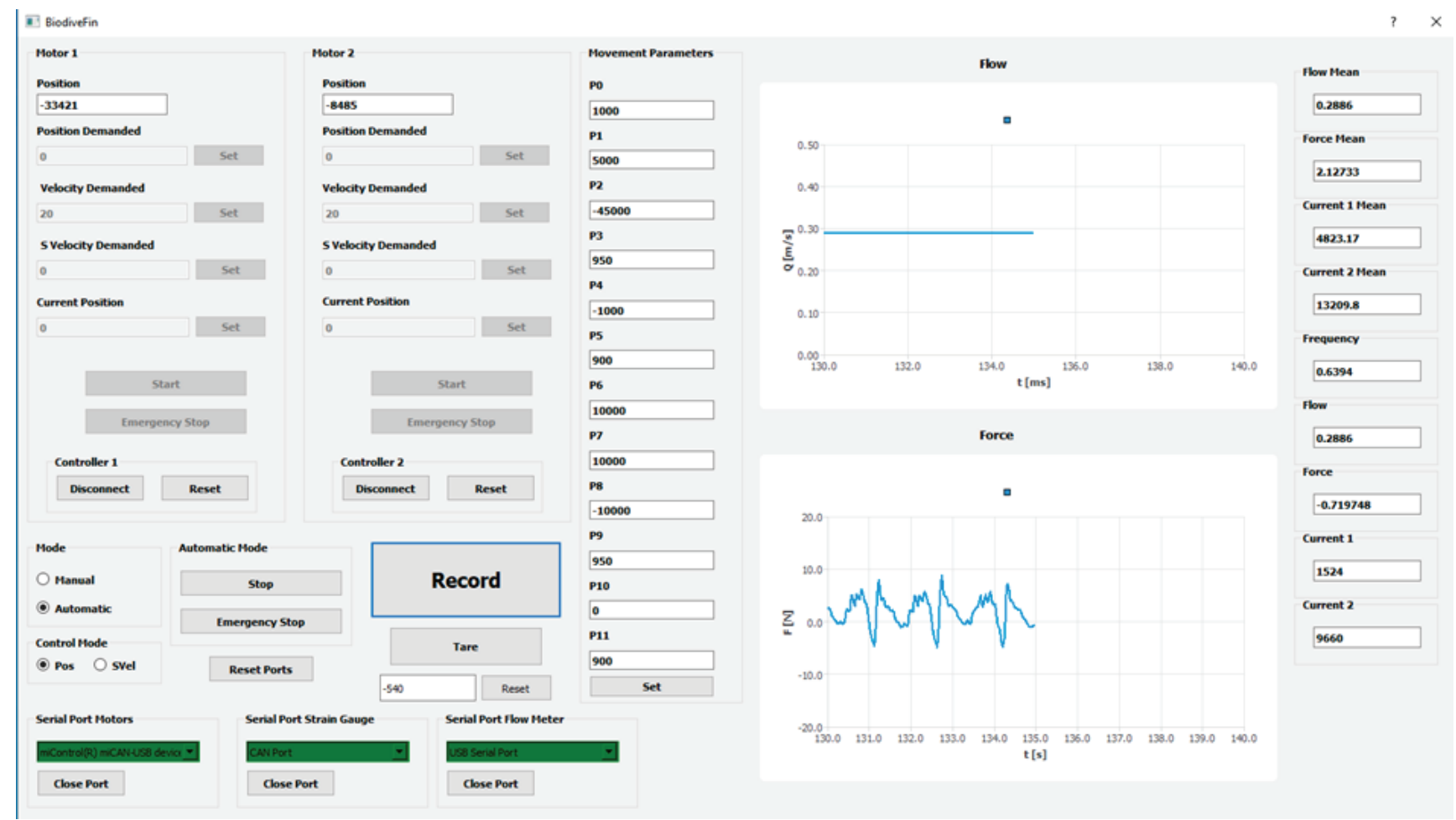

Fig. 6. Screenprint of the interface of the manipulator test stand with dynamic parameters of fins in operation [5]

- P4 - the target position, upper calf,

- P5 - the angular velocity of calf movement,

- P6 - the top position of the thigh in relation to the calf,

- P7 - the top position of the calf relative to the position of the thigh,

- P8 - the target position of the thigh in the lower position,

- P9 - the angular velocity of the thigh in the lower position,

- P10 - the target position of the calf in the lower position,

- P11 - the angular velocity of the calf in the lower position.

During the test, the next parameters were recorded and used for further analysis:

- The water speed $[\mathrm{m} / \mathrm{s}]$;

- The water stream $\left[\mathrm{m}^{3} / \mathrm{s}\right]$;

- The instantaneous value of the thrust force $[\mathrm{N}]$;

- An average value of the thrust force $[\mathrm{N}]$;

- An instantaneous value of the currents drawn by the motors no. 1 and $2[\mathrm{~mA}]$;

- An average value of the currents drawn by the motors no. 1 and $2[\mathrm{~mA}]$;

- The flipper frequency $[\mathrm{Hz}]$.

The interface of the manipulator test stand (Fig. 6), provides the ability to change the frequency of the manipulator's thigh and lower leg by adjusting the speed of movement and the values of the maximum deflections in the upper and lower position of both moving segments. The ankle joint is reflected in the form of a flexible element with a limited swing angle, and is determined in the indicated tags of ankle and toes measurements.

The same measurement conditions for all types of the fins can be obtained by adjusting the amplitude and the frequency as well as angular velocity using eleven parameters depicted [21]. Moreover, these parameters can be adapted to the results of the video of divers' limbs movement. The energy and kinematic parameters of the manipulator are archived automatically, and only the values of the traverse edge stroke of both tested fins were read visually.

\subsection{Plan of the experiment}

It was assumed that water is considered as an incompressible fluid with constant temperature, density $(\rho)$ and viscosity $(\gamma)$. The Reynolds number defined as a ratio of inertia to viscosity forces $\operatorname{Re}=\frac{L \cdot v}{\gamma}$ should be the same for all type of fins. That is why, for constant dimension of the manipulator leg, the measurements should be provided for 
the same range of fluid velocity. The towing power $\left(N_{h}\right)$ of the stationary manipulator with fin is proportional to the fluid velocity $(v)$ and shape dependent coefficient $(R)$, according to the equation (1):

$$
N_{h}=R \cdot v .
$$

For constant fluid velocity, the Strouhal number is used for characterizing the structure of the stream following the fin according to the following equation (2):

$$
S t=\frac{f A}{v},
$$

where:

$f$ - a flipper frequency;

$A$ - an amplitude of the fin trailing edge.

The horizontal thrust force component was measured directly with the use of strain gauge measurements system. Energy efficiency tests were designed to indicate the energy demand of the manipulator equipped with different types of fins. The energy input was proportional to the current value in the manipulator's electric motors $\left(N_{M 1}\right.$ and $\left.N_{M 2}\right)$. The electric power consumption was proportional to the force the fin works on the water, and defined as load factor $\tau_{T}$ characterizing the unit pressure $\frac{T}{S}\left[\frac{\mathrm{N}}{\mathrm{m}^{2}}\right]$. The active fin surface $A_{f}\left[\mathrm{~m}^{2}\right]$ presented in Table 1 has an impact on the maximal current restriction in servomotors. The efficiency was calculated as the ratio of average thrust $T_{\text {avg }}$ generated by the fin to the average power $P_{\text {avg }}$ consumed by artificial leg drivers. The average values were calculated for period of time proportional to the few leg interval with restriction to constant fluid velocity in water tunnel.

In the next stage of an analysis, the fin efficiency was calculated using Particle Image Velocimetry
(PIV) method, where the water inflow sped to fin $v$ and the water velocity at the trailing edge $v_{T E}$ wass be compared. This analysis may give more complex and detailed information about parameters impact on the fin efficiency.

Scenarios for recreational swimming were proposed, which corresponded to the most common behaviour of swimmers:

- Small Amplitude and Low Frequency of oscillation (SALF), typical for free speed swimming;

- Large Amplitude and High Frequency of oscillation (LAHF), analogy to fast diving;

- Large Amplitude and Low Frequency of oscillation (LALF), typical for making turns;

- Small Amplitude and High Frequency of oscillation (SAHF), typical fast swimming in a restricted area, e.g., caves, wracks, etc.

All experiments were carried out under the same simulated conditions for four models of float leg movement in the measuring channel. The swimmer motion patterns mentioned above were based on the motion of real swimmers identified using the optical system [12]. The motion patterns include time varying positions of hip and knee joints, i.e., changes of both an amplitude of motion oscillation of each joint and also a phase difference between motion oscillations of each joint.

The research on the dynamic features of two types of recreational fins was aimed at validating the kinematic parameters of movement and determining the dynamic and energetic characteristics of both fins. The tests were carried out for three representative swimming speeds, understood as constant water velocities in the water tunnel. Variable kinematic parameters performed by the programmable manipulator were the frequency of movement and upper and lower deflections. Graphic structure of the research plan is shown in Fig. 7. Each fin was examined for four patterns of motion: SALF,

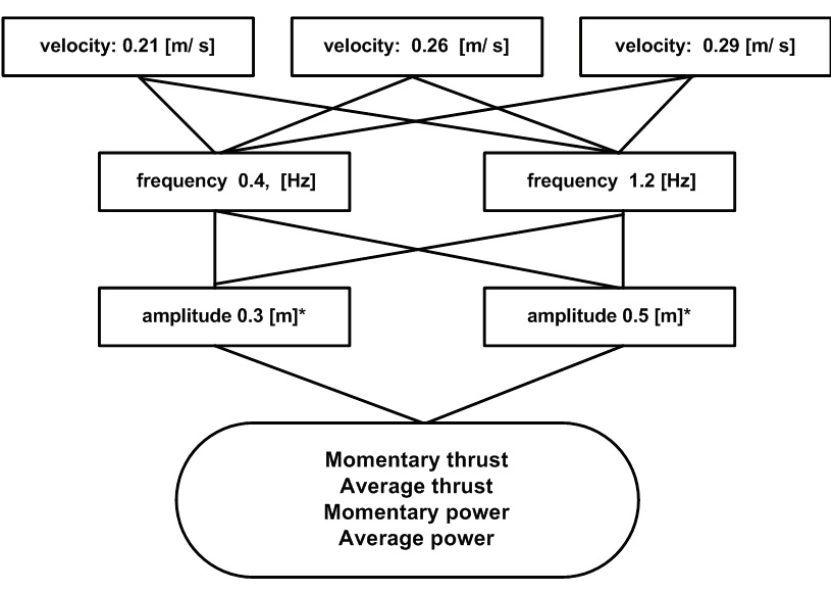

amplitude = foot deflections: * function (the fin length \& the fin flexibility)
Fig. 7. Graphic structure of the research plan 
Table 1. Geometric parameters of the fins

\begin{tabular}{|c|c|c|c|c|c|c|c|}
\hline Fins type & $\mathrm{B}$ & $\uparrow$ & 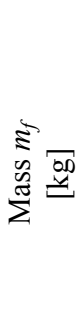 & 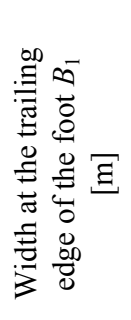 & 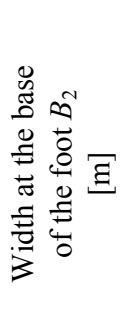 & 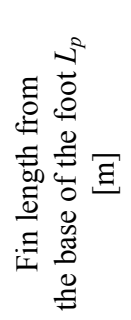 & 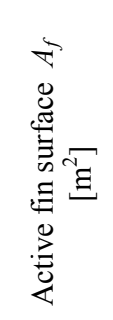 \\
\hline Aqualung Express Fin (AEF) & B & $\downarrow$ & 0.55 & 0.205 & 0.145 & 0.325 & 0.05687 \\
\hline Scubapro Jet Fin (SJF) & & & 1.7 & 0.235 & 0.2 & 0.3 & 0.06525 \\
\hline
\end{tabular}

SAHF, LALF and LAHF and each motion of the fin was tested for three different velocities of water flow inside the tunnel. To summarise, the following parameters of water flow and fin motion were used:

- 3 water flow velocities: $0.21,0.26$ and $0.29[\mathrm{~m} / \mathrm{s}]$,

- 2 frequencies of the fin motion: $0.4,1.2[\mathrm{~Hz}]$,

- 2 foot deflections: $0.3,0.5$ [m].

The obtained values of the kinematic and dynamic parameters were related to the dimensions of the fins illustrated in Table 1.

\section{Results}

All the tests were carried out for three constant velocities of water in the tunnel, provided by the electric motor - screw propeller set. In Figure 8, measurement results are provided in the form of the average values of produced thrust $\left(T_{\text {avg }}\right)$ and power consumed $\left(P_{\text {avg }}\right)$ by the examined fins oscillating with different parameters and in the absence of water flow with different velocities, while in Figs. 9 and 11 examples of changes in time of momentary thrust produced by the fins AEF and SJF were inserted.

Taking into consideration the average thrust $T_{\text {avg }}$ and corresponding average power $P_{\text {avg }}$ (Fig. 8) obtained for different motion patterns and different velocities of water flow in the tunnel, we can formulate the following conclusions:

- Increasing the amplitude and, to a lesser extent, the frequency of the fin oscillation resulted in an increase of the thrust produced by the fins;

- AEF produced larger thrust than SJF except during SAHF motion pattern. It can be caused by the fact that AEF has a larger active surface compared to SJF. Although, during motion with fast oscillation and small amplitude (SAHF), the more elastic fin with the larger active surface is not able to change effectively its attack angle effectively;

- AEF showed significantly lower power consumption for a small amplitude of the fin oscillation (SMLF, SAHF) at similar values of produced thrust, which indicates their higher efficiency;

- For the most frequently used recreational swimming motion pattern, i.e., LALF, AEF is characterized by the production of higher thrust at almost identical power consumption, especially for larger velocities of water flow in the tunnel;

- AEF achieved the highest value of Reynolds number, which is the result of its greater length.

The structure of the time course of the thrust produced by SJF indicates the symmetry of operation (Fig. 9), which means that in the work cycle, there are two active movements in the direction of the velocity vector. In contrast, AEF work is characterised by asymmetrical thrust (Fig. 10), which indicates the effects of flexible deflection in the dead upward movement of the fin.

Obtained results enable us to determine values of Strouhal number and fin efficiency illustrated accurately in Tables 2 and 3.

Considering values of Strouhal number for different motion patterns illustrated in Table 2, we can observe optimal conditions of the fin motion for SALF. Conditions of motion according to the patterns other than SASF differ from the conditions of free-swimming fish.

An analysis of the calculated fin efficiency based on the thrust load factor indicates a higher efficiency of AEF compared to SJF fins. The efficiency analysis (Table 3) was provided for large amplitude of the movement for both reference fins. 


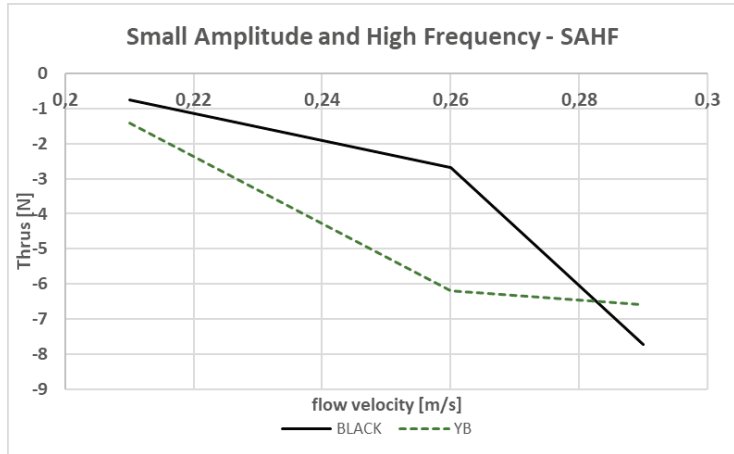

(a)

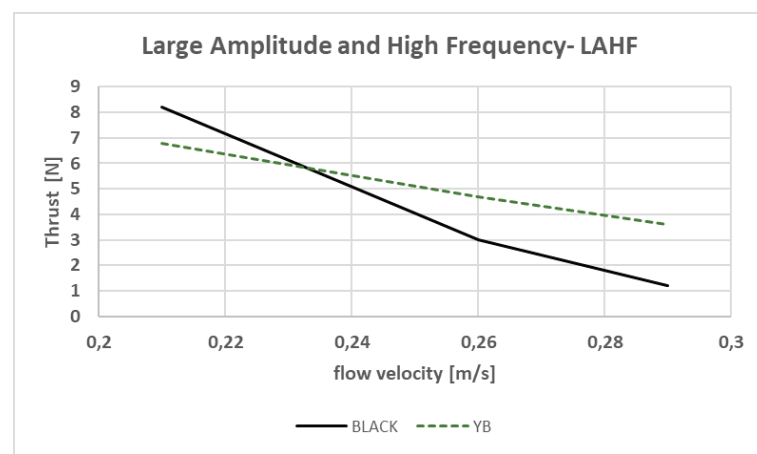

(b)

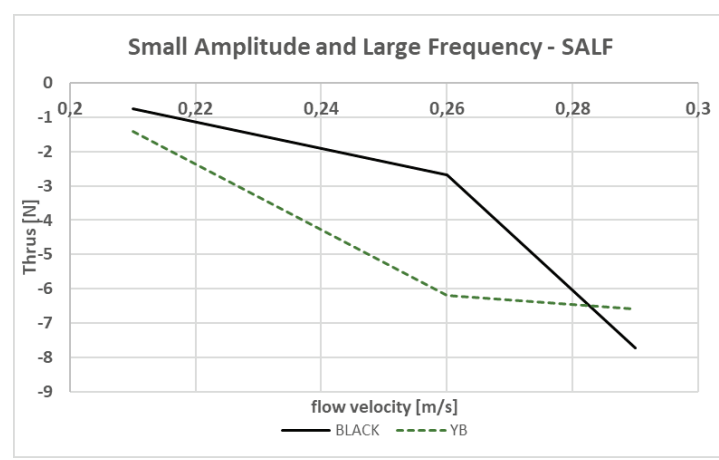

(c)

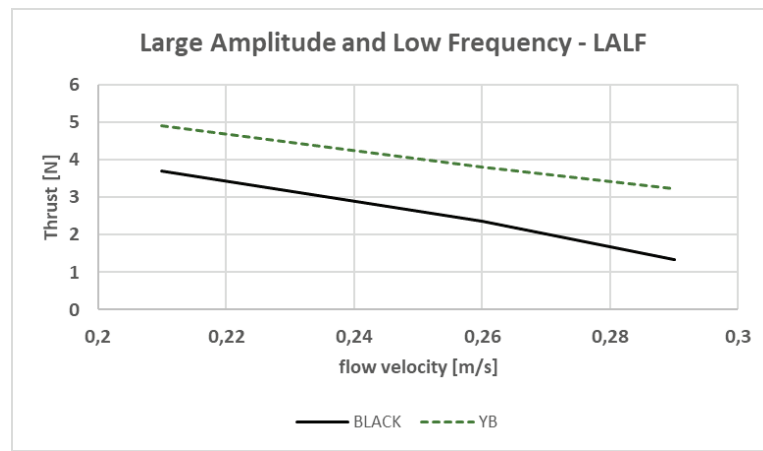

(d)

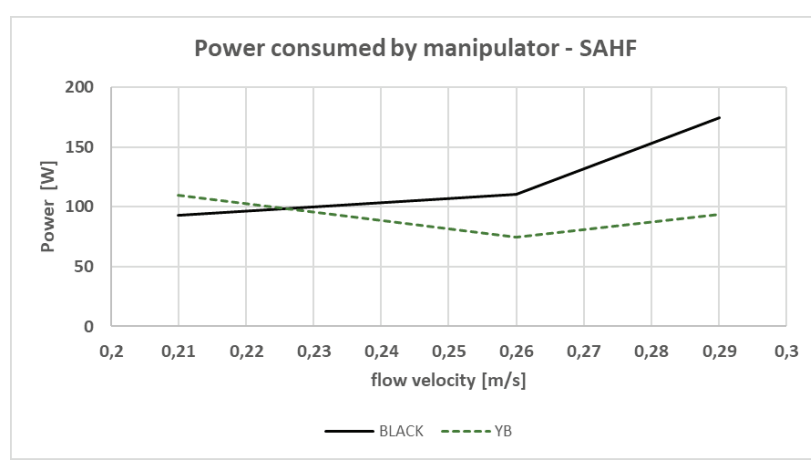

(e)



(f)

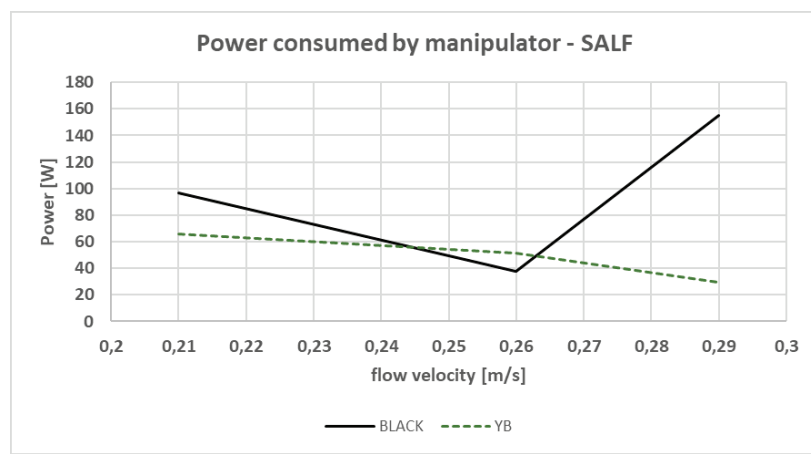

(g)

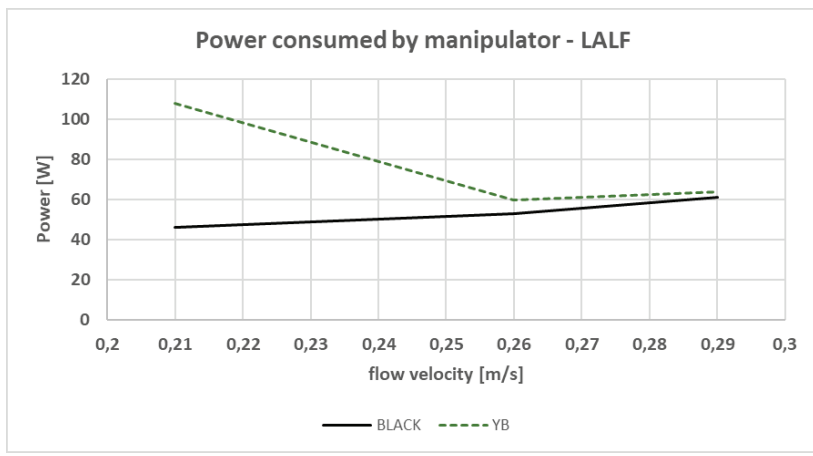

(h)

Fig. 8. Average thrust $T_{\text {avg }}$ produced by fins AEF and SJF oscillating with (a) SAHF, (b) LALF, (c) SALF,

(d) LALF and corresponding average power $P_{\text {avg }}$ consumed by the fins oscillating with (e) SAHF, (f) LAHF, (g) SALF, (h) LALF 
Thrust vs time, Jest Fin, $\mathrm{f}=14 \mathrm{~Hz}$, LAHF

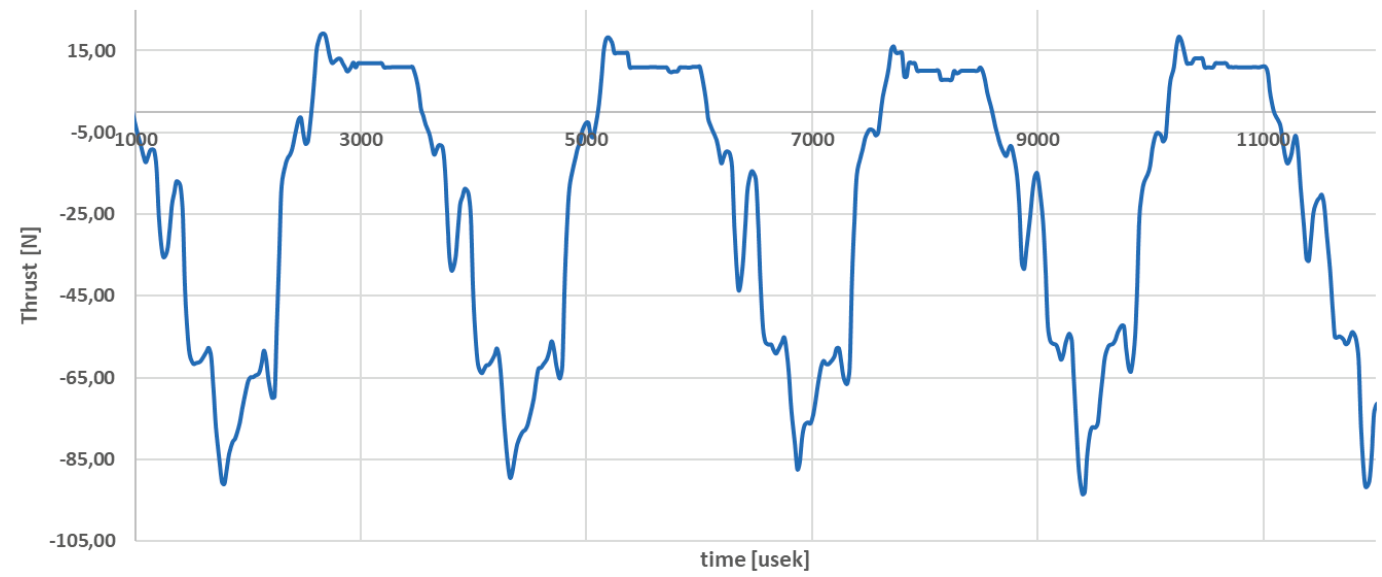

Fig. 9. Changes in time of momentary thrust $T$ produced by Scubapro Jet Fin oscillating with LAHF with water flow $0.26 \mathrm{~m} / \mathrm{s}$

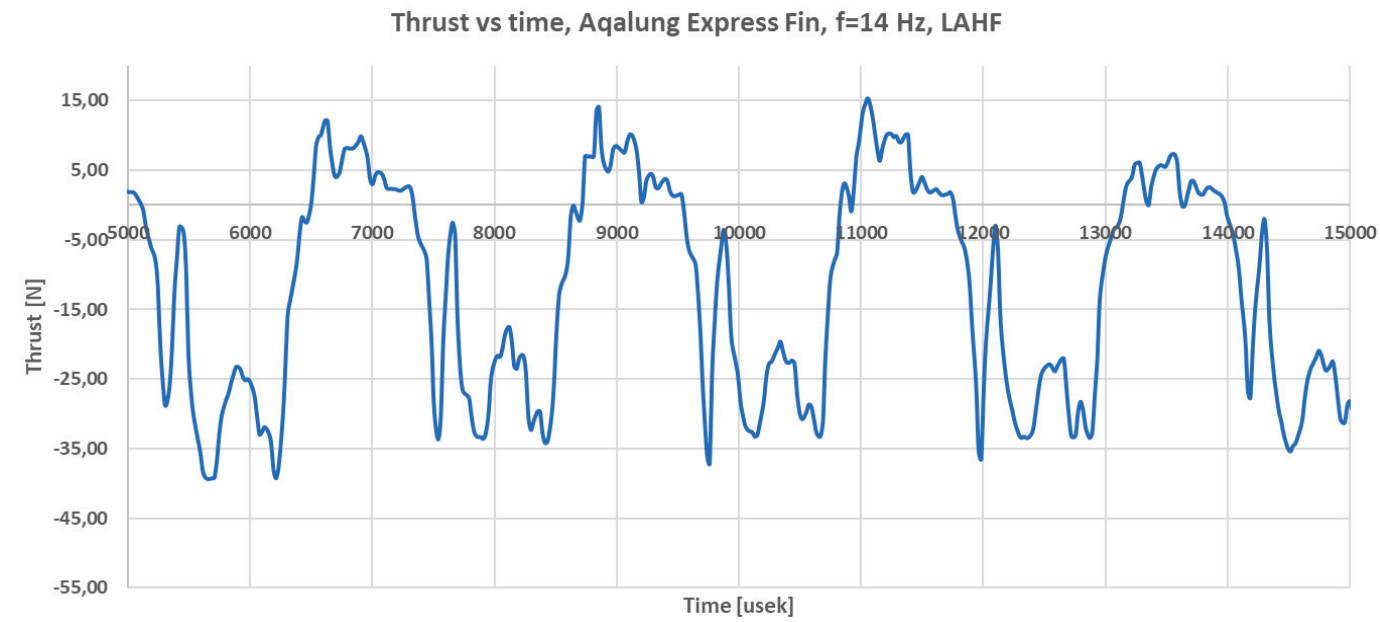

Fig. 10. Changes in time of momentary thrust $T$ produced by Aqualung Express Fin oscillating with LAHF with water flow $0.26 \mathrm{~m} / \mathrm{s}$

Table 2. Strouhal numbers for examined reference fins: $\mathrm{AEF}$ and SJF

\begin{tabular}{|c|c|c|c|}
\hline \multirow{3}{*}{$\begin{array}{c}\text { Parameters } \\
\text { of fin oscillation }\end{array}$} & \multirow{2}{*}{$\begin{array}{c}\text { Velocity } \\
\text { of water flow } \\
{[\mathrm{m} / \mathrm{s}]}\end{array}$} & \multicolumn{2}{|c|}{$\begin{array}{c}\text { Strouhal number }[-] \\
\text { for }\end{array}$} \\
\cline { 2 - 4 } & 0.21 & AEF & SJF \\
\hline \multirow{3}{*}{ SASF } & 0.26 & 0.6 & 0.68 \\
\cline { 2 - 4 } & 0.29 & 0.53 & 0.51 \\
\hline \multirow{3}{*}{ LASF } & 0.21 & 1.45 & 1.41 \\
\cline { 2 - 4 } & 0.26 & 1.18 & 1.02 \\
\cline { 2 - 4 } & 0.29 & 1.03 & 0.96 \\
\hline \multirow{3}{*}{ SALF } & 0.21 & 4.12 & 3.98 \\
\cline { 2 - 4 } & 0.26 & 2.89 & 3.02 \\
\cline { 2 - 4 } & 0.29 & 2.29 & 2.45 \\
\cline { 2 - 4 } & 0.21 & 2.09 & 2.02 \\
\cline { 2 - 4 } & 0.26 & 1.71 & 1.64 \\
\hline
\end{tabular}

Table 3. Fin efficiency for examined reference fins: $\mathrm{AEF}$ and SJF

\begin{tabular}{|c|c|c|c|}
\hline \multirow{3}{*}{$\begin{array}{c}\text { Parameters } \\
\text { of fin oscillation }\end{array}$} & \multirow{2}{*}{$\begin{array}{c}\text { Velocity } \\
\text { of water flow } \\
{[\mathrm{m} / \mathrm{s}]}\end{array}$} & \multicolumn{2}{|c|}{$\begin{array}{c}\text { Fin efficiency }[-] \\
\text { for }\end{array}$} \\
\cline { 2 - 4 } & 0.21 & AEF & SJF \\
\hline \multirow{3}{*}{ SALF } & 0.26 & - & - \\
\cline { 2 - 4 } & 0.29 & - & - \\
\hline \multirow{3}{*}{ LALF } & 0.21 & 0.65 & 0.6 \\
\cline { 2 - 4 } & 0.26 & 0.73 & 0.67 \\
\cline { 2 - 4 } & 0.29 & 0.82 & 0.72 \\
\hline \multirow{3}{*}{ LAHF } & 0.21 & 0.65 & 0.64 \\
\cline { 2 - 4 } & 0.26 & 0.76 & 0.69 \\
\cline { 2 - 4 } & 0.29 & 0.78 & 0.73 \\
\hline \multirow{3}{*}{ SAHF } & 0.21 & - & - \\
\cline { 2 - 4 } & 0.26 & - & - \\
\cline { 2 - 4 } & 0.29 & - & - \\
\hline
\end{tabular}




\section{Discussion}

In contrast to the objective evaluation of the fins, the divers often ranked the stiff fins better than their flexible counterparts [11]. Another result questioned was the reliability of air intake consumption by the divers as a cost of swimming energy for the different fin models [4]. Further, passively measured body resistance with fins [8] may be insufficient for dynamic analysis of the fin parameters. Hence, the decision to design and build a water tunnel with a one-to-one scale equipped with precise sensors. Besides the fin popularity in the market, their characteristic features emphasised and analysed earlier on informed the choice of commercial fins. The electric motors that provide an objective value of power consumption also drive the submerged artificial leg. At the same time, the thrust force is a direct measurement of the fluidstructure interaction phenomenon.

The method presented in this paper is based on measurement of thrust generated by manipulator moving with the same desired motion pattern. Therefore, it is devoid of human error and, subsequently, provides more reliable and independent results. While in [10], the results were achieved using twelve international level swimmers. Although, the swimmers were performing at a high level of swimming techniques (four of them were medalists in World Championship), the statistical analysis had to be provided. Also, the results presented in [15] differs due to different strategies of leg movements.

Taking the kinematics into consideration, the presented method has limitation, i.e., fin motion is controlled in an open-loop control system and in a vertical surface (changes of 2D coordinates of the hip, knee and ankle joints). On the other hand, every fin can be examined for exactly the same kinematic measurement conditions.

Active Drag Evaluation System used in [1] is based on Velocity Perturbation Method presented by [3] and used for active drag computation. In the case of force measurements [9], swimmers were linked via a non-elastic wire to a force sensor with a belt placed around the hip joint. As shown in [16], the drag coefficient for towed swimmers significantly decreases with swimming depth. Taking the active drag measurements presented in this paper into consideration, it can be observed that the mean value of active drag force is equal to $5.36[\mathrm{~N}]$ for Aqualung Express Fin and $3.20[\mathrm{~N}]$ for Scubapro Jet Fin. The presented measurements were made for Low Amplitude and High Frequency (LAHF), with a water velocity of $0.26 \mathrm{~m} / \mathrm{s}$.
The passive drag force [13] mainly depends on the shape while the active drag force depends on the amplitude and frequency. It was shown how the active drag force changes with time, in a range $52.06[\mathrm{~N}]$ for Aqualung Express Fin and in a range 108.34 [N] for Scubapro Jet Fin. This result can be compared with [10], where active drag for underwater swimming was $78.90[\mathrm{~N}]$ and for surface swimming was about 84.7 [N]. It is worth underlining that the thrust measurements presented in the paper have been performed in the liquid environment with flowing water, while the measurements included in [10] have been obtained for tethered swimmers. Moreover, it is worth emphasizing that in the present research, only one artificial leg was designed in order to reduce the impact of body shape on the fins parameters. Thus, a more detailed analysis can be carried out in terms of the shape of the fin and the influence of material parameters on the fin capabilities. On the other hand, this method does not undertake the problems of interaction between two fins.

Additionally, the active drag force is affected by swimming technique, which can be evaluated through Strouhal number and Froude efficiency. The Strouhal number is the tradeoff between amplitude and frequency that generates forward speed. Taking marine animals into account, the value of Strouhal number ranges from 0.25 to 0.40 [6], [7]. While the lowest value of Strouhal number for professional swimmers with highest velocities, highest efficiencies and lowest active drag equal to 0.34 has been obtained in [9]. In this research, the Strouhal number ranges from 0.51 to 4.12 with a mean value of 1.67 for Aqualung Express Fin and mean value of 1.63 for Scubapro Jet Fin. The efficiency analysis presented in [9] shows the tendency to peak (equal to $82 \%$ of Froude efficiency) at a narrow range of Strouhal numbers near 0.4. According to the presented method, the fin efficiency was calculated as a ratio of a power of consumed electric energy to the generated thrust. This presented proposition is due to the ambiguity in Froude efficiency calculation, and it means that it is difficult to calculate the ratio of the thrust power in comparison with total power. The efficiency calculation presented in this paper is based on electric power consumption measurements and direct method of thrust measurements. This proves a more convenient way to fin quality assessment. Froude efficiency equal to 1 means that the total power is equal to the thrust power, thus, the efficiency presented in this paper means that all the electric power is converted to the propulsion force. The Froude efficiency calculated for a crawl is equal to 0.42 for swimmer without fins and 0.50 for those with fins [20]. The fin efficiency calculated according to the 
proposed method starts with a minimum value of 0.6 for Scubapro Jet Fin with parameters of oscillation Large Amplitude Large Frequency (LALF) to a maximal value of 0.82 for Aqualung Express Fin. The high value of fin efficiency is primarily because the fin is a designed and optimized commercial products. It is expected that the newly designed fins are to be tested in the water tunnel, and the efficiency could be lower at the beginning. However, having it ready to use water tunnel with accurate measurement system as well as manipulator guarantying repeated kinematic and dynamic conditions, it will be possible to evaluate a new fin design and material parameters as well as different strategies of leg movements.

What is more, the vision system can be applied for verification of various fin swimming techniques. The vision system is currently capable of doing verification, but not to control at real-time in a closed-loop system. Further, it is unable to control the movement of an artificial leg because of the lack of software that can directly control the position of the artificial leg in the closed-loop.

According to the method, it is possible to have an analysis of the dynamics and efficiency associated with diving fins as a continuous system. In making use of a wide variety of input data, the fin construction optimization process is available for the different control algorithms obtained from a selected sample of the divers. The method consists of all technical data created for a dynamic system. Further, from the characteristics presented, the water speed may influence the selection of material and construction parameters of the fins.

\section{Conclusions}

The article presents a method for estimating the dynamics and efficiency of the fins. There was a method verification performed on the commercial fins, which is one of the most popular fins on the market. The discussion on the results obtained from the fins' operation seem to be comprehensive thanks to the presented test stand of the water tunnel. Although, the stand enables us to extend conditions of measurements, e.g., other motion patterns and other velocities of water flow in the tunnel.

The presented research results and the discussion of the literature lead to the following conclusions. The tests carried out with the use of a leg manipulator have an advantage over the results known in the literature, mainly in the repeatability of the experimental condi- tions and the accuracy of dynamic parameter measurements. The same applies to the laminar flow conditions of the water inflow provided in the tunnel. The presented tunnel lab stand can be used for testing other fins and small propellers as well as for training divers to dive in small spaces, e.g., wrecks or underwater caves. The authors of the paper also see weaknesses in their work. The first problem is the assessment of changes in the efficiency of the manipulator drive during one work cycle for different behaviours of swimmers motions. The second problem is that the efficiency of the manipulator's electromechanical drive is much lower than that of the fins hydromechanical one. Both problems definitely had an impact on the achieved results. For this reason, the authors made a lot of effort to ensure that the measurement conditions for the tested fins were identical.

The expectation is that the above would change after measuring the biomimetic fins, which are currently in the process of implementation.

\section{Acknowledgements}

The research presented in the paper was co-financed by the Polish National Centre of Research and Development within project no. POIR.01.01.01-00-0046/17 carried out in 2017-2020 years.

\section{References}

[1] Bideau B., Colobert B., Nicolas G., Le Guerroué G., Multon F., Delamarche P., Development of an active drag evaluation system (A.D.E.S.), [in:] J.C. Chatard (Ed.), Biomechanics and medicine in swimming $I X$, Publications de l'Université de Saint Etienne, France, 2003, pp. 51-56.

[2] Groh B.H., CiBis T., Schill R.O., EsKofier B.M, IMUbased Pose Determination of Scuba Divers' Bodies and Shanks, Conference paper, DOI: 10.1109/BSN.2015.7299376, https:// www.researchgate.net/publication/296827144, 2015

[3] Kolmogorov S., Duplisheva O., Active drag, useful mechanical power output and hydrodynamic force coefficient in different swimming strokes at maximal velocity. Journal of Biomechanics, 1992, 25, 311-318.

[4] Morris K.S., Osborne M.A., Shephard M.E., Jenkins D.G., SKINNER T.L., Velocity, Oxygen Uptake, and Metabolic Cost of Pull, Kick, and Whole-Body Swimming, Int. J. Sports Physiol. Perform., 2017 Sep., 12 (8), 1046-1051, DOI: 10.1123/ ijspp.2016-0322. Epub 2016, Dec 14, PMID: 27967275.

[5] MinaK G., Evaluation of the performance of free-diving fins, Sports Eng., 2004, No. 7, 153-158, DOI: 10.1007/BF02844053.

[6] Morawski M., Malec M., ZajaC J., Development of CyberFish - Polish Biomimetic Unmanned Underwater Vehicle BUUV, Applied Mechanics and Materials, 2014, 613, 76-82, https:// doi.org/10.4028/www.scientific.net/amm.613.76

[7] Morawski M., Malec M., Szymak P., Trzmiel A., Analysis of Parameters of Traveling Wave Impact on the Speed of Biomimetic Underwater Vehicle, Solid State Phenomena, 2013, 210, 273-279, https://doi.org/10.4028/www.scientific. net/ssp.210.273. 
[8] Nakashima M., Tanno Y., Fujimoto T., Masutani Y., Development of a Simulation Model for Swimming with Diving Fins, Proceedings, 2018, No. 2, 288, DOI: 10.3390/ proceedings 2060288 .

[9] Nicolas G., Bideau B., Colobert B., Berton E., How are Strouhal number, drag, and efficiency adjusted in high level underwater monofin swimming?, Human Movement Science, 2007, 26, 426-442.

[10] Nicolas G., Bideau B., A kinematic and dynamic comparison of surface and underwater displacement in high level monofin swimming, Hum. Mov. Sci., 2009 Aug., 28 (4), 480493, DOI: 10.1016/j.humov.2009.02.004. Epub 2009, Apr. 22, PMID: 19395109.

[11] Pendergast D.R., Mollendorf J., Logue C., Samimy S., Evaluation of fins used in underwater swimming, Undersea and Hyperbaric Medical Society, UHM, 2003, Vol. 30, No. 1, 55-71.

[12] Piskur P., Szymak P., SzNAJder J., Identification in a Laboratory Tunnel to Control Fluid Velocity, [in:] Bartoszewicz A., Kabziński J., Kacprzyk J. (eds.), Advanced, Contemporary Control, Advances in Intelligent Systems and Computing, 2020, Vol. 1196, Springer, Cham., https://doi.org/ 10.1007/978-3-030-50936-1_128.

[13] Piskur P., Szymak P., Flis L., SzNAJder J., Analysis of a Fin Drag Force in a Biomimetic Underwater Vehicle, OUR SEA: International Journal of Maritime Science and Technology, 2020, Vol. 67, No. 3, https://doi.org/10.17818/NM/ 2020/3.2

[14] Praczyk T., Szymak P., HożyŃ S., Applying Optical System to Model the Motion of Human Leg Moving in Water According to Swimming Style Crawl, Proceedings of 8th Inter- national Maritime Science Conference, Montenegro, 2019, 215-222.

[15] Rejman M., Siemontowski P., Siemienski A., Comparison of performance of various leg-kicking techniques in fin swimming in terms of achieving the different goals of underwater activities, PLoS One, 2020, Aug 3, 15 (8), e0236504, DOI: 10.1371/journal.pone.0236504, PMID: 32745109, PMCID: PMC7398542.

[15] Vennell R., Pease B., Wilson B., Wave drag on human swimmers, Journal of Biomechanics, 2006, 39, 664-671.

[16] Wojtкów M., Nikodem A.M., Biomechanics of diving: the influence of the swimming speed on the kinematics of lower limbs of professional divers, Acta Bioeng. Biomech., 2017, Vol. 19, No. 4, 117-125.

[17] Wylegala J., Schafer-Owczarzak M, Pendergast D.R., Optimization of fin-swim training for SCUBA Divers, UHM, 2007, Vol. 34, No. 6, 431-437.

[18] Zamparo P., Pendergast D.R., Termin B., Minetti A.E., How fins affect the economy and efficiency of human swimming, The Journal of Experimental Biology, 2002, Vol. 205, 2665-2676.

[19] Zamparo P., Pendergast D.R., Mollendorf J., Termin B., MinetTi A.E., An energy balance of front crawl, European Journal of Applied Physiology, 2005, 94, 134-144.

[20] Zamparo P., Froude efficiency in human swimming, Comparative Biochemistry and Physiology, Part A, Molecular and Integrative Physiology, 2008, Vol. 150, Issue 3, Supplement, ISSN 1095-6433, https://doi.org/10.1016/j.cbpa.2008.04.078. (http://www.sciencedirect.com/science/article/pii/S10956433 08001694). 